\title{
El uso de la expresión plástica en los museos de Bolonia
}

The use of the arts expression in the museums of Bologna

Carmen Gómez-Redondo*, Beatrice Borghi ${ }^{* *}$

\section{Resumen}

En el siguiente artículo se muestran los resultados de una investigación sobre el uso de técnicas plásticas en los programas educativos de los museos de la ciudad de Bolonia. Para ello se ha diseñado una herramienta específica para la recogida de datos sobre el uso de técnicas plásticas en los diseños educativos en los museos de la ciudad, siguiendo con los principios de investigación de corte cualitativo. Tras el análisis de los datos obtenidos los principales resultados que arroja el estudio se pueden observar cómo los museos incorporan las técnicas plásticas en sus diseños, pero principalmente técnicas tradicionales y con un sentido lúdico.

Palabras clave: Expresión plástica. Educación museal. Educación Patrimonial. Museos.

\begin{abstract}
The following article shows the results of an investigation on the use of plastic techniques in the educational programs of the museums of the city of Bologna. To this end, a specific tool has been designed to collect data on the use of plastic techniques in educational designs in the city's museums, following the principles of cualitative method. After analyzing the data obtained, the main results of the study can be seen how museums incorporate plastic techniques in their designs, but mainly traditional techniques with a playful sense.
\end{abstract}

Key words: Arts work. Museum education. Heritage education. Museums.

\section{Introducción}

Suárez, Gutiérrez, Calaf y San Fabián (2013) ya señalaban los numerosos estudios existentes sobre el público y la acción educativa en los museos, aunque consideran que quedan muchos aspectos que aún no han sido explorados. Faltan nuevas perspectivas de evaluación (San Fabián, 2014) para el reconocimiento de buenas prácticas y la obtención de información de calidad, pero para ello es esencial concebir el museo como un espacio para el aprendizaje Hooper-Greenhill (1998).

\footnotetext{
* Universidad de Valladolid - ORCID: 0000-0002-7636-0251 - Autora de correspondencia: mariacarmen.gomez.redondo@uva.es

** Università di Bologna • ORCID: 0000-0001-6716-7620.
}

Gómez-Redondo, C. y Borghi, B. (2020). El uso de la expresión plástica en los museos de Bolonia. CLIO. History and History teaching, 46, 188-201. https://doi.org/10.26754/ojs clio/clio.2020465270. Recibido: 28/5/2020. Aceptado: 20/9/2020. 
Desde la perspectiva educativa, las aportaciones de García (1994) y Fontal (2008) son esclarecedoras y nos permiten vislumbrar una evolución en los diseños educativos museales, obligados en la actualidad, por la creación de nuevos contextos educativos, como es el incuestionable caso de Internet Tejera (2013) y (Vicent, 2016).

A este respecto, podríamos decir, que, si bien en un primer momento los departamentos de educación de los museos importaron los modelos educativos de la escuela, los modelos actuales dan cuenta de una emancipación educativa del ejemplo formal, siendo ahora éstos los referentes educativos en innovación y adaptación a los nuevos contextos e intereses de los participantes (Borghi, 2005).

Desde que Lowenfeld y Brittain (1980) hablaran del potencial autoexpresivo de las artes, esta ha sido una de las cualidades indisociables de la Educación Artística, que ha ido enriqueciéndose desde entonces. La educación artística, hace tiempo que dejó de centrarse en la reproducción sin sentido de técnicas plásticas (Aguirre, 2000) y desde luego, ya no consiste en aquellas manualidades por el día del padre. Caminamos ahora hacia modelos pragmatistas (Aguirre, 2000), modelos globales (Juanola y Calbó, 2004) y modelos patrimoniales (Fontal, 2013), en los que los procesos, la reflexión crítica, la experiencia y la emoción se entrelazan para formar una experiencia de aprendizaje plena (Borghi, Francisco y García-Pérez, 2015). Estas perspectivas y procesos específicos de una educación artística son extrapolables a los procesos de patrimonialización y generan sus propias aportaciones, dando al proceso de patrimonialización una tonalidad propia. Analizamos aquí algunas de esas aportaciones, comenzaremos desde las más conocidas hacia las más específicas del ámbito, ¿Qué pasa cuando introducimos actividades de Educación Artística en nuestros diseños patrimoniales?

- Percepción como algo lúdico y distendido: Los aprendizajes experimentales, de manera general, son percibidos por los participantes (sobre todo los escolares) como aprendizajes de más fácil adquisición, generalmente no requieren de un silencio o agrupaciones restrictivas, sino más bien al contrario, se necesita intercambio de ideas, diversidad de agrupaciones, etc. Lo que hace que la percepción de este tipo de actividades sea más positiva. 
- Aporta actividades activas, participativas y experienciales a los diseños de educación patrimonial (Calaf, 2009): las actividades artísticas no pueden ser pasivas o simplemente cognitivas, sino que precisan de la manipulación y de la activación de los procesos creativos, por lo que, por definición, van a aportar estas cualidades a los diseños patrimoniales.

- Interpretación y subjetivación: La educación Artística va más allá de la ejecución de técnicas, permite la expresión, la aportación de la propia mirada y la proyección de lo subjetivo, por tanto, permite una mayor subjetivación del patrimonio. A través de la creación de una obra subjetiva creamos significados subjetivos para el patrimonio, llegamos a la apropiación simbólica (Gómez-Redondo, 2013). El arte nos abre un canal, el incluso una autopista desde el patrimonio a la persona (Gómez-Redondo y Fenoy, 2014).

- Cambio de lenguaje: La Educación Artística no es solo expresión y ejecución, gran parte de su significatividad pasa por la reflexión y el diálogo. Sin embargo, esta reflexión y diálogo no se hace por los canales habituales de comunicación, se abren nuevos canales y por tanto cambian los mensajes y las ideas que detonan en el proceso dialógico. Lo artístico permite expresar lo inefable ¿cómo son esas emociones y sentimientos que provoca el patrimonio y no se pueden explicar con palabras? ¿Cómo son los discursos y diálogos que se producen a través de este nuevo canal? Se abre ante nosotros un nuevo universo de ideas y diálogos en torno al patrimonio.

- Generación de nuevos patrimonios (Fontal, Gómez-Redondo y Pérez, 2015): Las autoras proponen que las nuevas obras (individuales o grupales) creadas a partir del trabajo en torno al elemento patrimonial pueden, además, convertirse en un nuevo patrimonio, tal vez efímero, que ayuda a generar nuevos vínculos con el patrimonio de referencia, llevándonos a la siguiente cuestión.

- Creación de redes de relaciones: Desde la propia creación se crean redes de vínculos, con el elemento patrimonial de referencia, con nuevos elementos... hablamos por tanto de un sistema patrimonial. En el que la educación artística puede actuar como material fundamental para generar estas relaciones, una especie de mielina u hormigón. 
- Generación de nuevas identidades (Fontal, Gómez-Redondo y Pérez, 2015): La creación de una nueva obra, supone la proyección de la subjetividad individual o del grupo, supone por tanto una reformulación de la propia identidad al incorporar ese patrimonio dentro, ayudando a visibilizar y a "hacer un hueco" en el conjunto identitario.

- Metáforas: La metáfora es el gran aliado en los procesos de patrimonialización, que son tan abstractos y subjetivos. Las metáforas artísticas nos permiten comprender y poner en común algunos de esos procesos, añadiendo, además, nuevos significados a partir de la relación con la metáfora.

- Visibilización de los propios procesos de patrimonialización e identización: La metáfora y el proceso creativo en sí nos permiten visibilizar y compartir estos procesos, ser conscientes de los cambios que ocurren a nivel subjetivo y permitir un diálogo en torno a esto, ayudando así a la empatía, como sentimiento fundamental en la creación de identidades colectivas.

Sin embargo, las investigaciones en torno al uso de la Educación Artística en la Educación Patrimonial no aporta muchas esperanzas, la inferencia desde la teoría a la práctica no sucede y la Educación Artística en los diseños patrimoniales (que no son específicos del patrimonio artístico) queda, normalmente, reducida a un uso lúdico o como una reproducción de técnicas.

\section{Metodología}

A través de este trabajo se pretende conocer y comprender la presencia y el uso de las técnicas plásticas en los diseños educativos de los museos de la ciudad de Bolonia. Para ello se parte de un diseño de investigación de corte cualitativo. El diseño de la investigación sigue las fases y la lógica de los modelos cualitativos de investigación (Flick, 2015): recogida y análisis de datos y replanteamiento de la investigación en base a los datos obtenidos. 
Los resultados obtenidos en este proceso serán el punto de inicio para posteriores investigaciones que se centren en el análisis y evaluación en base a los resultados obtenidos.

Siguiendo a Arnal, Rincón y Latorre (1994) en el planteamiento de los objetivos de un estudio de casos, este trabajo tiene como finalidad:

- Describir y analizar los casos referentes para así completar la información obtenida en la fase anterior.

\section{Muestra}

El universo de programas educativos incorporados al estudio se compone de un total de 29 museos. En base a la variable 1 (V1) titularidad del museo, se observan 14 museos de la Universidad de Bolonia, gestionados por el Ateneo de la Universidad. 12 museos de titularidad pública municipal, 2 museos privados, 1 Museo de titularidad estatal.

Respecto a la Variable 2 (V2) categoría de patrimonio, estos museos se clasifican en: 4 están dedicados a la historia, 3 a arquitectura, 3 al patrimonio industrial, 4 al arte, 1 es un museo arqueológico, 11 son museos de ciencias, 1 a la música y 2 son museos etnográficos.

La titularidad de los museos va a ser una cuestión esencial respecto a la capacidad de respuesta a la herramienta de recogida de datos, ya que al ser gestionados por la misma administración se responde una única vez a la herramienta. Finalmente se ha obtenido información de 10 museos de los 29 museos contactados.

Respecto a la (V1) en los museos que finalmente han participado, 3 museos dependen del Comune, es decir, son de titularidad pública municipal; 6 gestionados por el Ateneo de la Universidad y finalmente 1 privado. Los datos obtenidos en relación con la (V2), el $60 \%$ de los participantes son museos de ciencias, un $20 \%$ museos de arte, un $10 \%$ de tecnología y otro $10 \%$ de Historia.

Finalmente, de los participantes, el $90 \%$ tiene un proyecto educativo y tan solo un $10 \%$ no lo tiene. 


\section{Diseño del instrumento}

Se somete la muestra a un estudio descriptivo a través del análisis de los datos obtenidos. Para ello partiremos de un instrumento específico (I1) (Ver anexo), diseñado ad hoc a partir de la consulta de bibliografía especializada sobre investigación y evaluación en museos (Suárez, Gutiérrez, Calaf y San Fabián, 2013), (Gómez-Redondo, Calaf y Fontal, 2016). Este instrumento se divide:

1. Descripción sociodemográfica del instrumento con 6 ítems de respuesta múltiple y respuesta dicotómica. El ítem número 4 condiciona la respuesta del resto del cuestionario, que en caso de ser negativa termina con un 5 ítem con respuesta múltiple. En caso de respuesta positiva se continúa con el cuestionario hasta finalizarlo.

2. Dimensión relacionada con las técnicas plásticas empleadas consta de 3 ítems de respuesta múltiple, respuesta dicotómica que conduce a una breve respuesta abierta.

3. Dimensión relacionada con los materiales empleados consta de 5 ítems de respuesta dicotómica.

4. Dimensión relacionada con los espacios donde se ubican las actividades formativas consta de 3 ítems de respuesta dicotómica.

5. Dimensión relacionada con el tiempo empleado para la realización de las actividades formativas consta de 3 ítems de respuesta múltiple.

6. Dimensión relacionada con el público al que se destinan las actividades formativas consta de 4 ítems de respuesta dicotómica y respuesta múltiple.

7. Dimensión relacionada con el uso didáctico de las técnicas plásticas empleadas consta de 7 ítems de respuesta dicotómica y respuesta múltiple.

8. Dimensión relacionada con sentido educativo de las técnicas plásticas consta de 4 ítems de respuesta dicotómica y respuesta múltiple.

El instrumento se ha traducido al italiano y se ha diseminado junto con una carta de presentación y unas instrucciones para su cumplimentación. Para facilitar la cumplimentación el cuestionario se ha adaptado al formato de formulario de la 
plataforma gratuita google forms. Que además de registrar y almacenar las respuestas a los cuestionarios ofrece un tratamiento de los datos estadístico/descriptivos de las respuestas.

\section{Resultados}

En relación con el primer cuerpo de preguntas, relacionadas directamente con aspectos generales de la acción educativa, los resultados sobre el tipo de público al que se destinan las propuestas educativas apuntan a una mayor atención al público escolar, con un claro predominio de la escuela primaria (Gráfico 1):

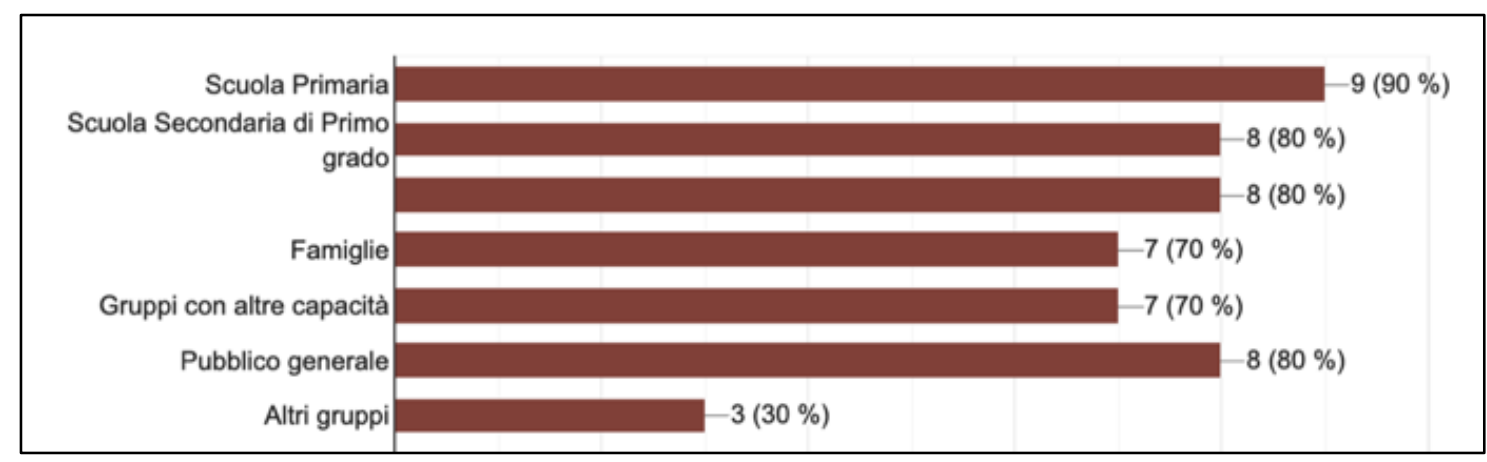

Figura 1. Colectivos a los que se dedican las propuestas educativas.

Estos programas educativos, en el $80 \%$ de los casos se realizan durante todo el año por un departamento educativo propio del muso, solo el $10 \%$ lo oferta de manera esporádica y en un $30 \%$ es realizado por empresas externas al museo.

Introduciéndonos en aspectos específicamente artísticos, el $70 \%$ de los museos que han respondido al cuestionario usa las técnicas artísticas en su oferta educativa, un dato realmente interesante si tenemos en cuenta ninguno de ellos son específicamente museos de arte. Del 30\% restante que no emplea técnicas artísticas las principales causas aludidas son: que los contenidos del museo no tienen relación con las artes (100\%) y que los contenidos presentados son de carácter conceptual $(50 \%)$, entendemos por tanto que sería necesario un estudio más profundo relacionado con las creencias sobre las aportaciones educativas de la expresión 
artística en los museos, para llegar a conocer estos casos que no emplean las técnicas artísticas por causas más de tipo educativo que infraestructural.

Entre las técnicas artísticas más empleadas destacan el collage, el ensamblaje como técnica escultórica y por debajo, el dibujo, la pintura y el moldeado. En menor medida el grabado, los medios audiovisuales, el modelado, las técnicas artesanales y la performance. Finalmente, la talla, la fotografía, la instalación y el netart destacan como las técnicas menos empleadas. Cabe destacar que las técnicas menos empleadas son las que precisan de mayores medios materiales: cámaras fotográficas, ordenadores con software específico, grandes espacios, etc.

Uno de los museos ha especificado, dentro del espacio reservado a la respuesta abierta, la escritura visual como técnica empleada.

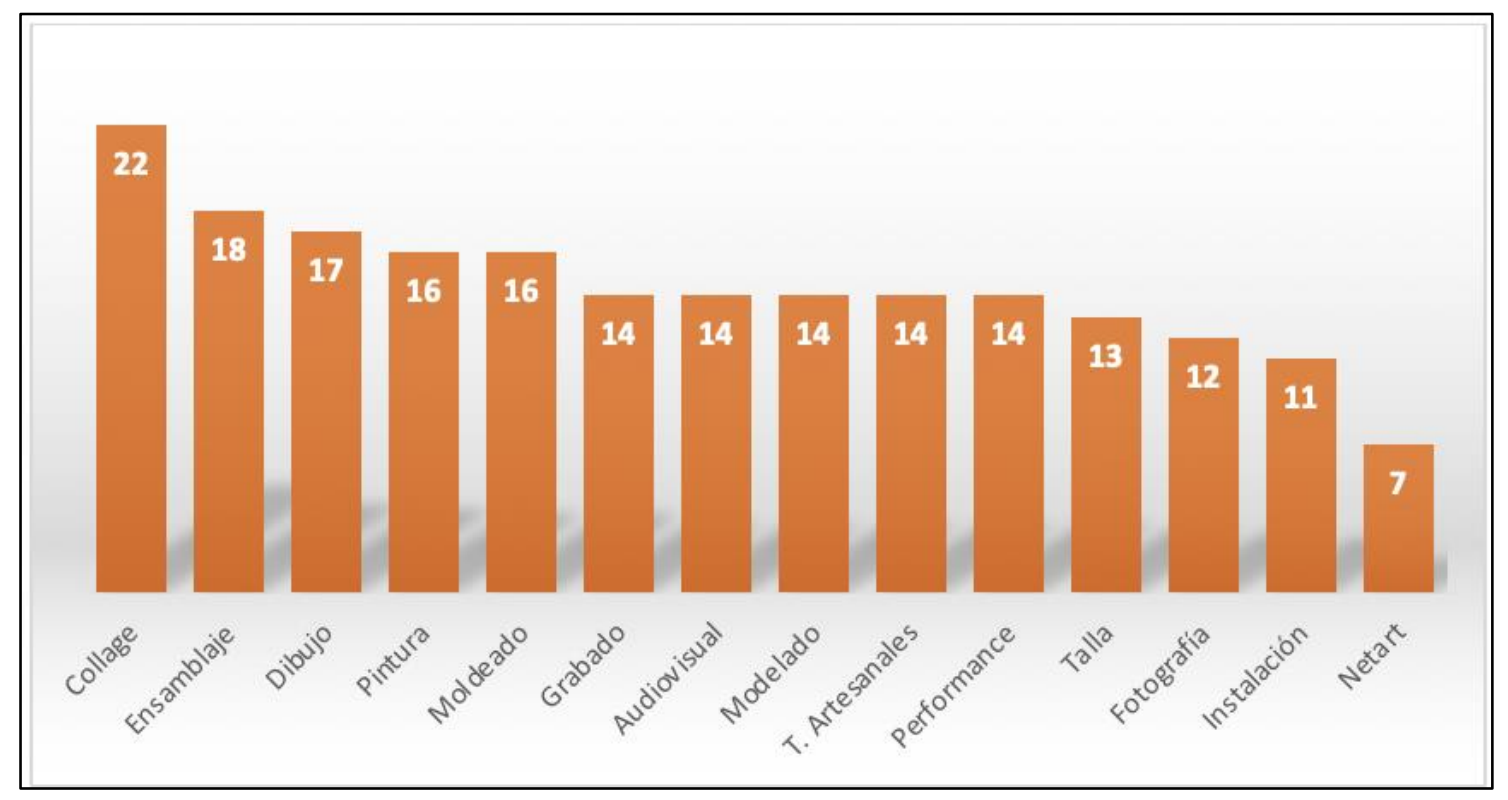

Figura 2. Técnicas artísticas empleadas por orden de uso.

Los ítems 7 y 8 del cuestionario, sobre el procedimiento para emplear estas técnicas, de manera general, dejan ver que se emplean las técnicas plásticas desde un punto de vista contemporáneo, en el que se mezclan diversas técnicas dentro de una misma obra. En menor medida se emplean procedimientos desde un paradigma moderno, es decir, ejecutando la técnica con distintos materiales a los originales y finalmente en raras ocasiones se afronta desde un paradigma académico, reproduciendo la técnica 
de manera tradicional. Además, un $85,7 \%$ emplean más de una técnica dentro de la misma sesión y en los programas en los que se utiliza mayoritariamente, son en los dedicados a la escuela primaria, en los de fin de semana y en los que tienen un carácter lúdico, es decir, las técnicas plásticas se emplean en varios programas de los museos participantes.

En los ítems relacionados con el empleo de los materiales los resultados señalan que casi todos los museos tienen un almacén para los materiales a emplear $(85,7 \%)$, que estos materiales no siempre están directamente relacionados con los materiales presentes en la colección (66,7\%), aunque resulta llamativo que un $33,3 \%$ se ciña exclusivamente a los materiales presentes en la colección. Además, los materiales utilizados son variados en un $85,7 \%$ de los casos y en el $100 \%$ se oferta variedad de material en la misma sesión. Los resultados son menos positivos cuando se hace referencia a materiales e infraestructuras más específicas ya que en el $71,4 \%$ de los casos, el museo no cuenta con material específico como caballetes, tórculos, etc. Además, en ese mismo porcentaje tampoco cuentan con infraestructuras adecuadas como ventilación, puntos de agua, luz... y un $57,1 \%$ no tiene elementos adecuados para un trabajo cómodo de los participantes (mesas, sillas, etc.)

En el recurso temporal observamos que un 57,1\% programa actividades que duran más de $2 \mathrm{~h}$, un $28,6 \%$ entre 1 y $2 \mathrm{~h}$ y finalmente un $14,3 \%$ diseña sesiones de menos de $1 \mathrm{~h}$. Dentro de esta temporalización, las actividades de creación ocupan la mitad del tiempo en el $100 \%$ de los casos. Respecto de la percepción del tiempo de creación/expresión en la actividad, el $85,7 \%$ cree que es suficiente y el $14,3 \%$ cree que es mucho tiempo.

En relación con los tipos de público, en el $85,7 \%$ de los casos existen diseños específicos para cada tipo de público y se adaptan a este, además en el $100 \%$ de los diseños las técnicas plásticas son adaptadas a las necesidades de los participantes. Respecto a las agrupaciones generadas para el desarrollo de las actividades de creación/expresión son generalmente $(71,4 \%)$ en pequeños grupos de 2 -3 personas y en un $28,6 \%$ de los casos en gran grupo de más de 10 personas. Además, este tipo de agrupaciones se realiza en un $85,7 \%$ en base a las necesidades del grupo y los perfiles a los que generalmente suele atender esta diversificación es a los grupos escolares y familias. 
El sentido didáctico del uso de las técnicas plásticas se entiende principalmente para obtener una valoración de la actividad (por ejemplo: dibuja lo que más te ha gustado de la actividad) y para interpretar la colección. En menor proporción aparece el sentido didáctico relacionado con obtener una valoración de la colección y fomentar el gusto por la expresión y los procesos de creación.

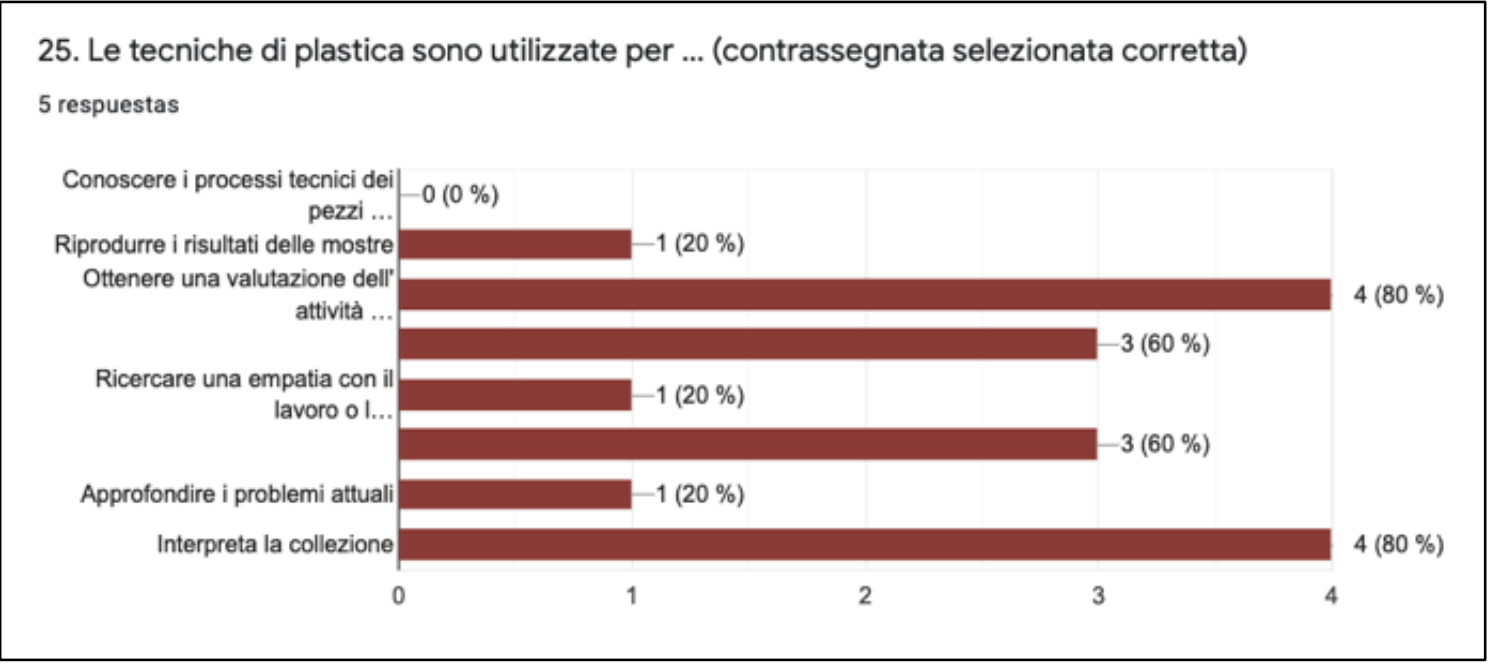

Figura 3. Sentido didáctico del uso de técnicas plásticas.

Este uso valorativo de las técnicas plásticas tiene también relación con el momento en el que se usan dentro del diseño de la actividad ya que en un $85,7 \%$ de los casos las técnicas plásticas se emplean al finalizar la actividad y solo en un $14,3 \%$ de los casos se emplea durante toda la actividad.

Sin embargo, los resultados obtenidos de las actividades de creación/expresión no son simples productos ya que un $85,7 \%$ de los museos considera que sí que usan las obras realizadas por los participantes como recurso educativo dentro de la propia actividad. Sin embargo, en el $57,1 \%$ de los casos estas obras no son expuestas y en el mismo porcentaje no son expuestas cerca de la exposición de referencia, aunque resulta alentador que esto sí que se haga en el $42,9 \%$ de los casos restantes. Este porcentaje se invierte en la cuestión relativa a la reflexión y valoración respecto de las propias creaciones y en un $57,1 \%$ sí que se realiza este proceso reflexivo.

En relación con el sentido educativo de los diseños, el $85,7 \%$ de los museos contemplan objetivos relacionados con la creación y la expresión dentro de sus 
diseños y respecto al sentido de la expresión/creación dentro del diseño podemos observar que de manera generalizada se emplea para conseguir una visión positiva y lúdica de la actividad y divertir, por debajo de estas dos intenciones aparecen la generación de una visión crítica, la visibilización de procesos de aprendizaje y la generación de vínculos. Finalmente, la menor de las intenciones es hacer terapia.

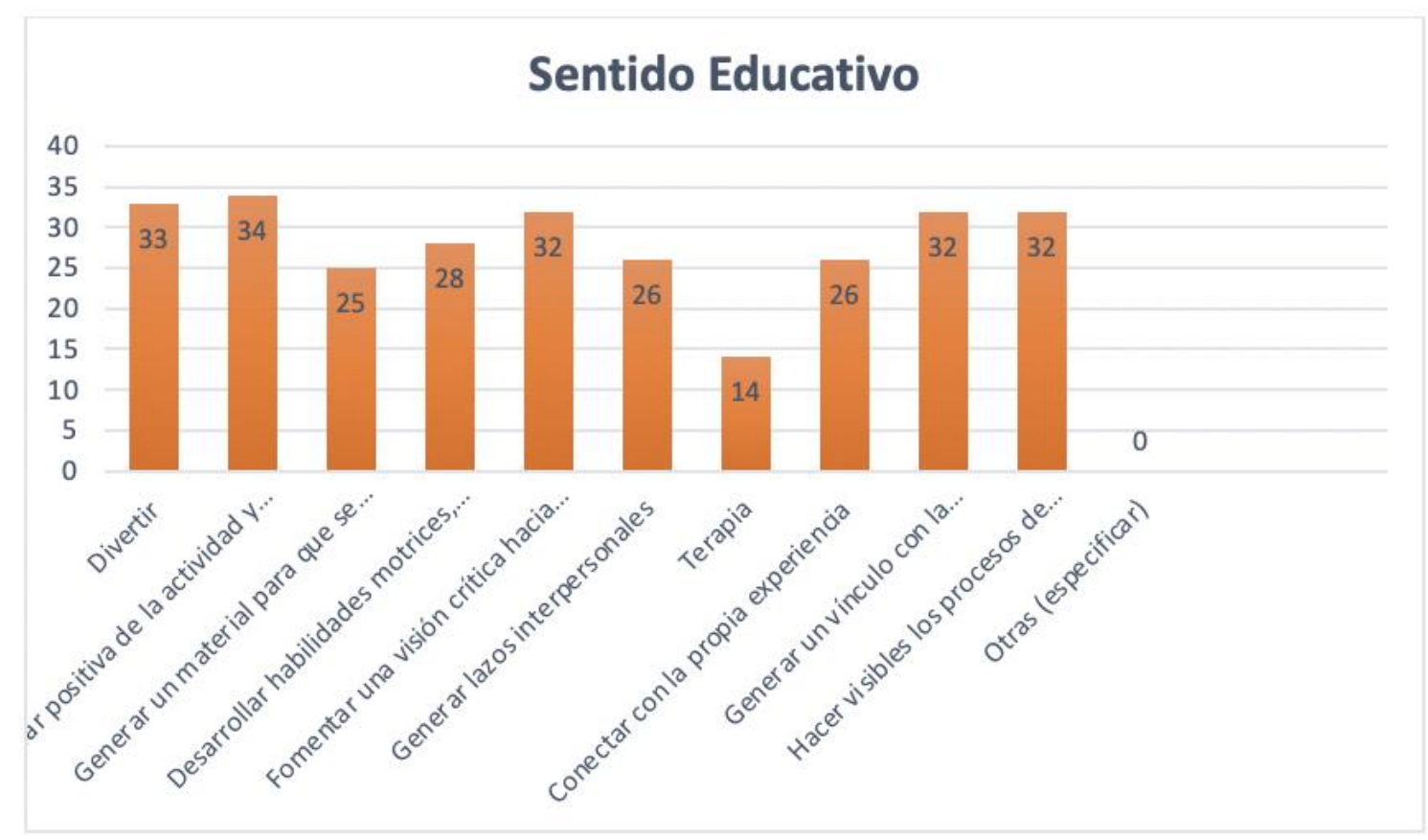

Figura 4. Gráfico 4. Sentido educativo del uso de técnicas plásticas.

Finalmente, respecto de las temáticas trabajadas en las actividades, el $85,7 \%$ de los museos trabaja otros temas socialmente de interés a través de la colección como y el $100 \%$ trabajan de manera coordinada con los centros educativos.

\section{Discusión y conclusiones}

En base al objetivo marcado para este trabajo, se considera que se ha llegado a realizar un primer análisis descriptivo de la didáctica en los museos de la ciudad de Bolonia, para ello se ha creado un instrumento específico de fácil acceso para obtener la mayor cantidad de datos de manera eficiente. Ha este respecto el instrumento ha 
respondido de manera positiva a su finalidad y además abre nuevas cuestiones para la profundización de la investigación.

A la vista de los resultados aportados resulta muy positivo el hecho de que museos que no están centrados en patrimonio artístico empleen las técnicas plásticas en sus diseños educativos. Sin embargo, las técnicas más empleadas suelen ser más tradicionales, tal vez por la necesidad de mayores infraestructuras por parte de otro tipo de técnicas (esto se podría valorar haciendo un estudio de correlatividad) o por la formación de los profesionales de los museos implicados. Además, como se ha podido observar, las actividades plásticas siguen ocupando una pequeña parte del diseño educativo y su empleo se concentra en la parte final de la actividad.

Resultan muy interesantes los datos aportados sobre el sentido didáctico y educativo del uso de técnicas plásticas en el diseño educativo. Respecto al sentido didáctico, es decir, para qué se usan las técnicas plásticas en la acción educativa, las respuestas que más predominan son las relacionadas con la interpretación de la colección y para obtener una valoración de la actividad, pero además resulta interesante saber que en ningún caso se emplea para reproducir las técnicas empleadas en la colección lo que contrasta con los resultados obtenidos por en investigaciones relacionadas pendientes de publicación.

Respecto al sentido educativo del empleo de técnicas plásticas las respuestas hacen referencia a un uso centrado en la generación de una experiencia positiva y la diversión, pero también en la generación de una visión crítica y en la creación de un vínculo con el patrimonio. Estos principales resultados nos llevan a pensar que el uso de técnicas plásticas está muy afianzado en los diseños educativos de Bolonia y que en muchos casos avanzan hacia un uso significativo en la acción educativa y no como simple acción lúdica, aunque ésta sigue estando muy presente en los diseños. En relación con esto, resulta de interesante cómo la acción educativa en relación con el uso de las técnicas plásticas tiene puntos de conexión con algunos principios de los modelos educativos propuestos (Aguirre, 2000 y Fontal 2013) en tanto en cuanto la experiencia se formula como una parte necesaria en el proceso de aprendizaje y el vínculo con el patrimonio es una de las finalidades del uso de las técnicas plásticas. 
De cara a futuras investigaciones resultará interesante profundizar en los casos cuyas respuestas apuntan hacia estos resultados. También resultaría de interés profundizar en los resultados obtenidos de estas acciones educativas a través de estudios de público.

\section{Referencias}

Aguirre, I. (2000). Teorías y prácticas de educación artística. Ensayo para una revisión pragmatista de la experiencia estética en educación. Octaedro.

Borghi, B. (2008). Un patrimonio di esperienze sulla didattica del Patrimonio. Pàtron.

Borghi, B., Francisco, F. y García Pérez O.M. (2015). Novi cives: cittadini dall'infanzia in poi. Pàtron.

Calaf, R. (2009). Didáctica del patrimonio: epistemología, metodología y estudio de casos. Trea.

Flick, U. (2015). El diseño de investigación cualitativa. Morata.

Fontal, O. (2008). Hacia una educación artística "patrimonial". En I. Aguirre (Coord.), El acceso al patrimonio cultural. Retos y debates (pp. 31-66). Cátedra Jorge Oteiza.

Fontal, O. (2013) Estirando hasta dar la vuelta al concepto de patrimonio. La educación patrimonial: del patrimonio a las personas (pp. 23-44), Trea.

Fontal, O., Gómez-Redondo, C., y Pérez, S. (2015). Didáctica de las artes visuales en la infancia. Paraninfo

García, A. (1994). Didáctica del museo. El descubrimiento de los objetos. Ediciones de la Torre.

Gómez-Redondo (2013). Procesos de patrimonialización en el arte contemporáneo. Diseño de un Artefacto artístico para la investigación (Tesis doctoral). Universidad de Valladolid.

Gómez-Redondo, C y Fenoy, B. (2014). El origen de los procesos de patrimonialización: la afectividad como punto de partida. EARI. Educación artística: revista de investigación, 5, 66-80. https://dialnet.unirioja.es/servlet/articulo?codigo=485959

Gómez-Redondo, C., Calaf, R. y Fontal, O. (2016). Colección "Roser Calaf" de recursos didácticos textuales para museos y sitios de patrimonio: análisis y valoración desde la perspectiva de la educación patrimonial. Revista de humanidades, 28, 85-114. http://revistas.uned.es/index.php/rdh/article/view/16495

Hooper-Geenhill, E. (1998). Los museos y sus visitantes, Trea.

Juanola, R. Y Calbó, M. (2004). Hacia modelos globales en educación artística. En R. Calaf y O. Fontal (Coords.), Comunicación educativa del patrimonio: referentes, modelos y ejemplos (pp. 105-136). Trea.

Latorre, A., Del Rincón, D. y Arnal, J. (2005). Bases metodológicas de la investigación educativa. Ediciones experiencia.

Lowenfeld, V. y Brittain, W.L. (1980). Desarrollo de la capacidad creadora. Kapelusz.

Stake, R. E. (2006). Evaluación comprensiva y evaluación basada en estándares. Barcelona: Ariel. 
Suárez, M. A., Gutiérrez, S., Calaf, R. y San Fabián J. L. (2013). La evaluación de la acción educativa museal: una herramienta para el análisis cualitativo. Clío, 39, pp. 1-45. http://clio.rediris.es/n39/articulos/Calaf.pdf

Tejera, C. (2013). Investigación didáctica: la cibermuseografía didáctica como contexto educativo para la enseñanza y el aprendizaje del patrimonio. Estudio de páginas web educativas de museos virtuales de arte. Clío, 39. http://clio.rediris.es/n39/articulos/Tejera.pdf

Vicent, N. (2016). Uso de tecnología móvil en educación patrimonial. Evaluación del programa Zarautz en tus manos. Enseñanza de las ciencias sociales: revista de investigación, 16, 67-79. https://www.raco.cat/index.php/EnsenanzaCS/article/view/334518 